\title{
A Fabry-Perot Spectrometer for High-Resolution Spectros- copy and Laser Work
}

\author{
Klaus D. Mielenz, Robert B. Stephens, and Karl F. Nefflen
}

\begin{abstract}
A Fabry-Perot spectrometer with a piezoelectric spacer was constructed to record with a recorder, or display with an oscilloscope, the fine structure of the Haidinger fringes. The spectrometer is of the fixed spacer design, which provides great stability of adjustment. It was used to record the Zeeman splitting of the green line of $\mathrm{Hg}^{193}$, as well as to exhibit, on the oscilloscope screen, the multimode output signal of a He-Ne gas laser.
\end{abstract}

\section{Introduction}

Modern advances in microwave techniques permit the detection and direct analysis of gigacycle frequencies. Optical frequencies, however, lie in the teracycle range and so, are still orders of magnitude beyond the state of that art. Optical methods, therefore, remain the most direct approach to analyze such frequencies as are encountered in laser work. Among them, of course, the Fabry-Perot interferometer or spectrometer is the foremost choice.

Essentially, a photoelectric Fabry-Perot spectrometer is a Fabry-Perot interferometer of variable optical plate separation $n t$, with a phototube viewing the center of the Haidinger ring pattern through a small circular diaphragm. As only the central portion of the ring pattern is used, it represents a linear frequency filter. With any change of $n t$, therefore, the phototube scans through the Airy intensity distribution. The phototube output thus obtained, and properly displayed as a function of $n t$ on a chart recorder or oscilloscope screen, is a reproduction of the intensity structure within the interference rings.

\section{The Jacquinot and Tolansky Spectrometers}

The first photoelectric Fabry-Perot spectrometer was built by Jacquinot and Dufour [1]. ${ }^{1}$ They enclosed an etalon, of fixed physical plate separation $t$, in an air-tight chamber and obtained a continuous change of $n t$ by varying the pressure $p$ inside it. With $p$ slowly varying linearly with time, a graph of intensity distribution versus frequency is obtained on a $Y$-axis recorder.

The Jacquinot spectrometer has been much used to record the fine and hyperfine structures of spectrum lines; compare, for instance, reference [2].

Another type of the spectrometer is due to Tolan-

1 Figures in brackets indicate the literature references at the end of this paper. sky and Bradley [3]. They employed a Fabry-Perot interferometer in which one plate is oscillated through a distance of the order of $\lambda / 2$ by means of a mechanical vibrator. The phototube output signal is fed into the $Y$-axis input of an oscilloscope and the horizontal sweep synchronized with the vibrator. A reproduction of the ring structure is then displayed on the screen.

Recently, Herriot [4] has used the Tolansky spectrometer, with confocal mirrors, and a permanent magnet and moving coil to oscillate one mirror, to detect laser modes.

In the Jacquinot spectrometer, high frequency noise is easily suppressed by limiting the frequency response of the recorder and recording at low speeds. Since the light source must remain stable within at least the recording period, unstable light sources cannot be analyzed. Adiabatic effects, on the other hand, prohibit a fast scan and, thus, the display of the signal with an oscilloscope.

The Tolansky spectrometer, as compared hereto, readily analyzes unstable light sources. However, a broader frequency pass-band is required to produce an undistorted pattern on the screen, and this will result in an undesirable display of noise. The mechanical vibrator does not lend itself easily to a sufficiently precise, slow continuous scan. The use of the same instrument with a recorder does not appear to be of merit, therefore.

In the design of conventional Fabry-Perot interferometers, a fixed spacer has long proved to be of great advantage [5]. A piece of fused quartz tubing, optically parallel at both ends and with the two mirrors optically contacted to it, has been found, in this laboratory, the most practical and simplest design. Such etalons have been shipped long distances without any need for readjustment at their point of destination. Jacquinot's instrument does use a fixed spacer. The oscillating spectrometers, as described, do not share this advantage, and are liable to have less stability.

The electrical control of the scan in Tolansky's spectrometer, on the other hand, is more convenient and versatile than a pressure control. 


\section{The New Fabry-Perot Spectrometer}

The new spectrometer to be described here combines the advantages of the Jacquinot and Tolansky designs, and is simpler in construction and handling than either. It can readily be used with either a recorder or an oscilloscope, is of the fixed spacer design, and is controlled entirely electrically by the simplest of means.

The etalon used (fig. 1) essentially consists of a piece of lead zirconate-titanate (Clevite piezoelectric ceramic PZT-4) tubing of 2.0 in. outside and $1.6 \mathrm{in.}$ inside diameter, with the mirrors contacted to its ends. The tube is silver-plated on its inside and outside. The voltages required to provide length changes are applied through wires soldered to the inside and outside of the ceramic tube.

The ceramic used yielded a linear expansion of about $0.5 \mathrm{~m} \mu$ per volt per inch. The voltage required to scan the etalon through its range without overlap, for visible light and a $3-\mathrm{in}$. tube, is of the order of $100 \mathrm{v}$. Since the ceramic is primarily a capacitive circuit element, the only current drawn is a changing current. A d-c voltage to provide a continuous linear expansion or contraction can, therefore, be obtained with a simple filtered power supply or with batteries. An a-c voltage to provide oscillations can be taken from the 60-cycle power line with a variable transformer. These characteristics make the ceramic easy to use.

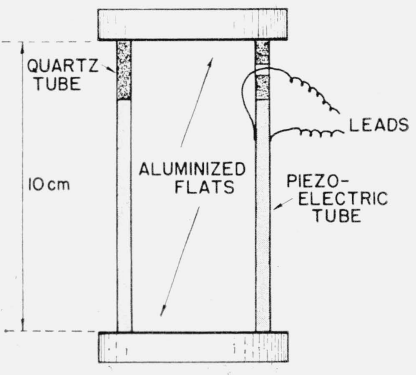

Figure 1. 10-cm etalon with piezoelectric spacer.

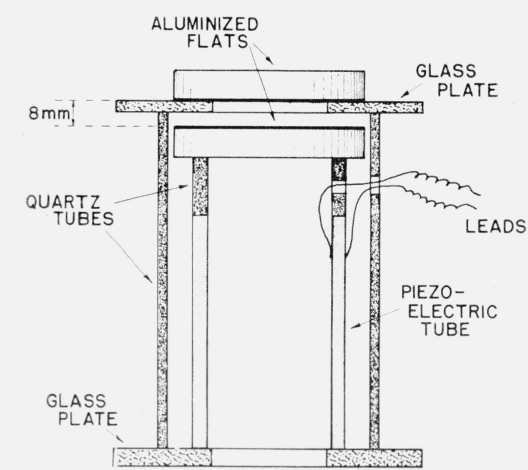

FIGURE 2. 8-mm etalon with piezoelectric spacer.
Another design, which was used to achieve a shorter plate separation with the 3 -in. tube at hand, is shown in figure 2. It might also prove advantageous when, for small spacings or long wavelengths, the higher voltages required by the other design tend to be impracticable. In both cases, flat mirrors were used, but it is planned to also use confocal plates for further experiments.

Although both etalons were used in an exploratory manner only, with the components held together by wax, and with aluminum mirrors of not more than 75 percent reflectance, the results obtained were very promising. The etalons were easy to adjust and maintained their adjustment. With d-c and a-c voltages high enough to provide a scan through five fringes, the expansion of the ceramic was found to be linear and reproducible. It did not affect the parallelism of the etalon at any time.

The etalons were employed in an upright manner, with a pentaprism at each end to deflect the light by $90^{\circ}$, figure 3 . A collimating lens of $199 \mathrm{~mm}$ focal length was used in front of, and a telescope lens of $275 \mathrm{~mm}$ focal length behind the etalon. The aperture of the diaphragm at the center of the ring pattern varies with the length of the etalon and was in each case chosen to yield optimum resolution.

With the $\mathrm{Hg}^{193}$ lamp, as employed in some of the experiments described below, a combination of Wratten filters was used to isolate the green line.

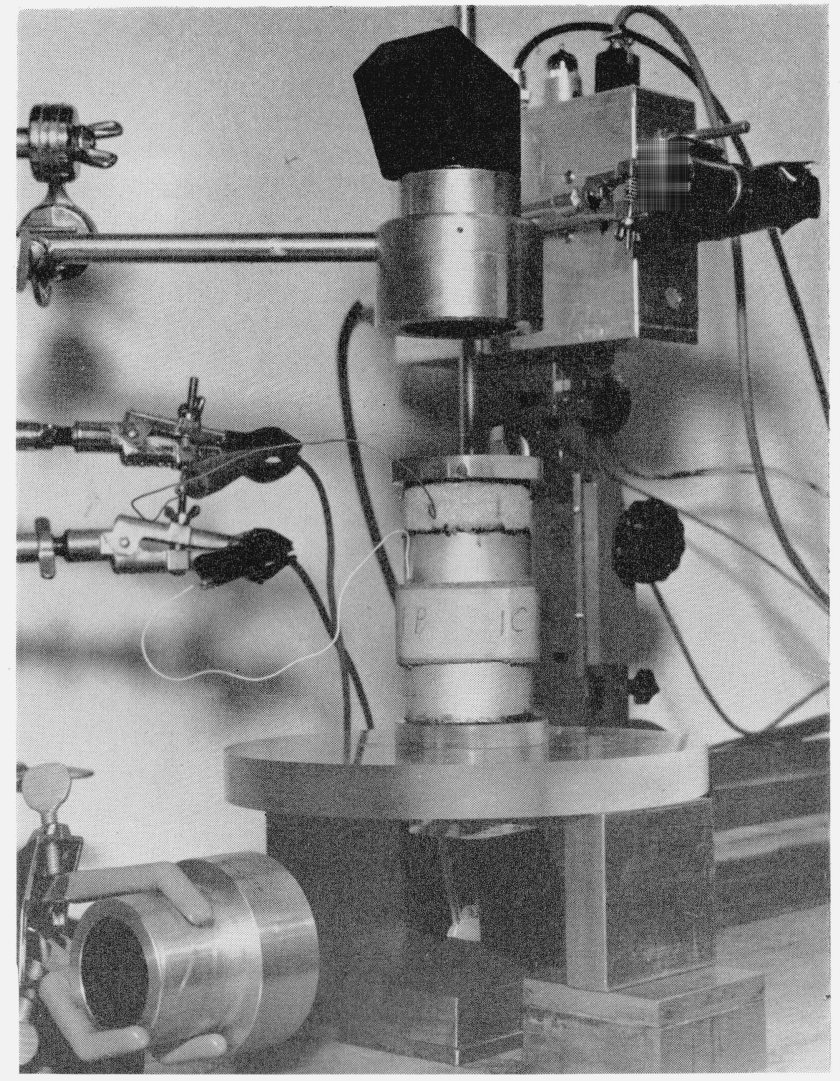

FIgURE 3. 10-cm etalon with lenses, pentaprisms, dixphragm, photomultipler, and cathode follower. 
A Fabry-Perot interferometer with continuous piezoelectric scanning was already described by Dupeyrat [6]. Being altogether different in design, it did not show much promise at the time.

\section{Experimental Results}

The new Fabry-Perot Spectrometer was employed in two ways, in a setup schematically shown in figure 4.

\subsection{Recording Spectrometer}

With a slowly linearly increasing or decreasing d-c voltage at the ceramic, the instrument represents the analog of the Jacquinot spectrometer. The voltage was supplied by a filtered full-wave power supply whose output of $410 \mathrm{v}$ was applied across a 10-turn precision $300 \mathrm{k} \Omega$ potentiometer driven by a $32 \mathrm{v} \mathrm{d}-\mathrm{c}, 1 / 3 \mathrm{rpm}$ motor. The resulting variations in the output signal of the RCA 1P21 photomultiplier were recorded versus time on a potentiometer-type recorder. Recording time was about $10 \mathrm{~min}$ per interference fringe.

The record reproduced in figure 5 shows the transverse, normal Zeeman splitting of the $5461 \AA \mathrm{Hg}^{198}$ line. It was obtained with a magnetron magnet of approximately 2200 oersteds applied to a watercooled $\mathrm{Hg}^{198}$ lamp excited by a microwave oscillator. The $8 \mathrm{~mm}$ etalon of figure 2 was used.

Figure 5a shows two rings with no magnetic field applied. In figure $5 \mathrm{~b}$, the line is broadened due to the field, and one can vaguely see the beginning resolution of a triplet. In figure $5 \mathrm{c}$, the $\pi$-component of the triplet is isolated by insertion of a Polaroid filter. Figure 5d shows the $\sigma$-components obtained after rotating the Polaroid by $90^{\circ}$.

The advantage of the recorder method lies, as mentioned, in the removal of noise. This is illustrated by contrasting figure 5 a with figure 6 , in which the unsplit $\mathrm{Hg}^{198}$ line is shown as obtained on the oscilloscope screen by the method described below.

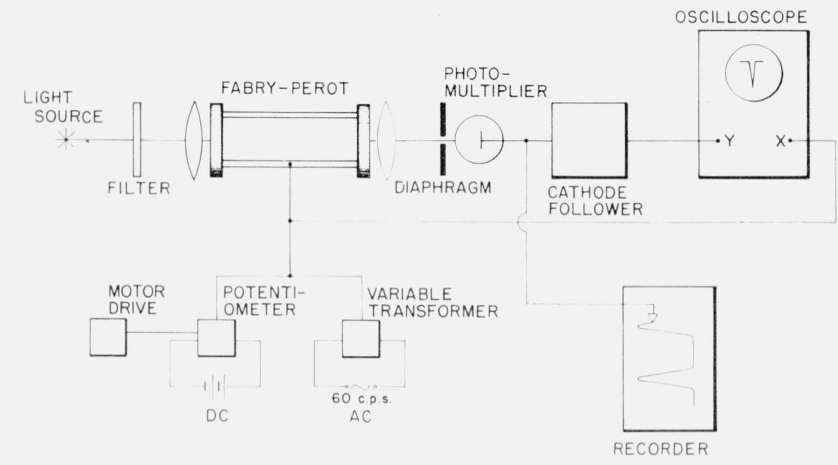

FIGURE 4. Block diagram of spectrometer with recorder and oscilloscope.

Due to a low signal-to-noise ratio, the oscilloscope pattern in figure 6 exhibits a strong photomultiplier noise which, however, is largely eliminated in the recorded pattern of figure $5 \mathrm{a}$.

\subsection{Oscillating Spectrometer}

With a 60-cycle a-c voltage at the ceramic and at the $X$-axis input of the oscilloscope, and the photo-

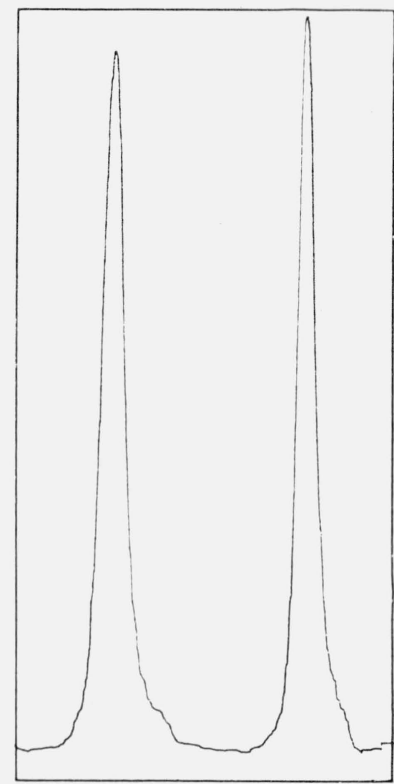

a

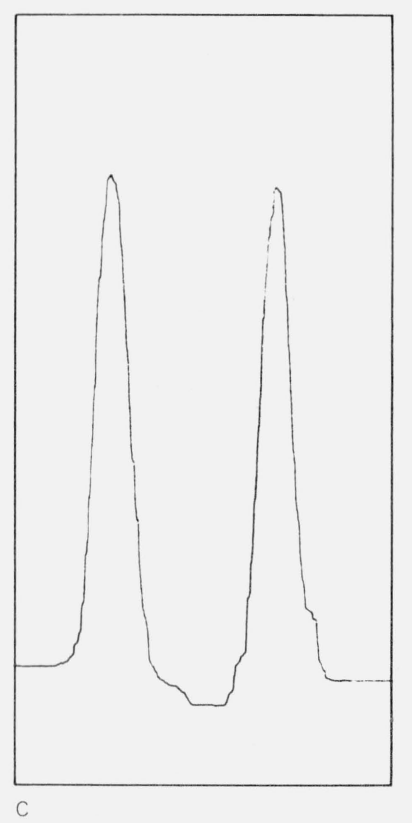

Figure 5. Normal Zeeman effect of green line of $\mathrm{Hg}^{198}$. (a) Without field; (b, c, d) with field, (b) all components, (c) $\pi$-component, (d)

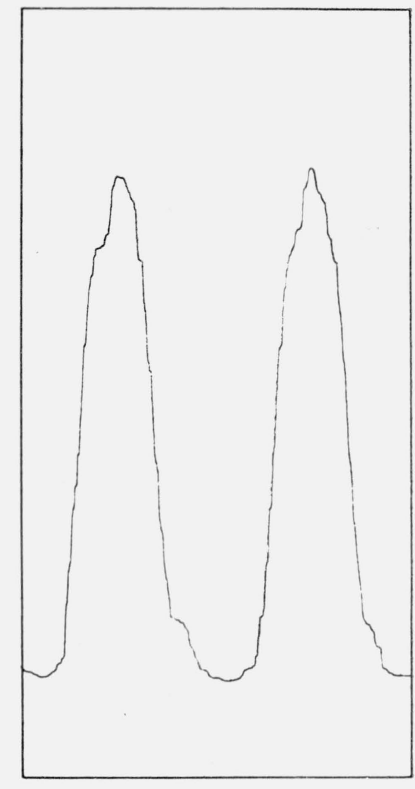

b

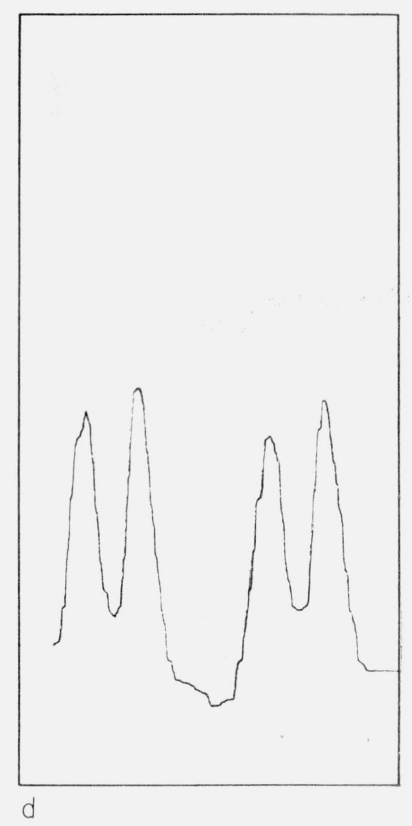




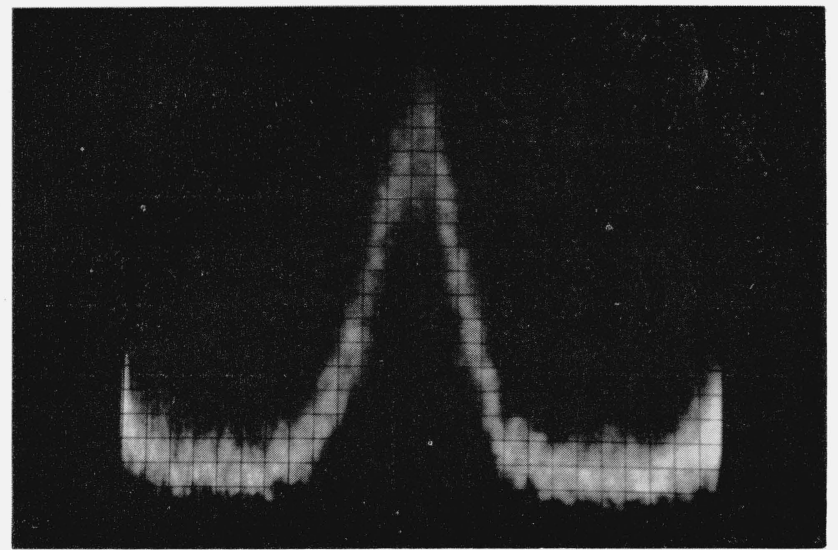

Figure 6. Oscilloscope pattern of green line of $\mathrm{Hg}^{1: 8}$, obtained at low signal-to-noise ratio. tube output fed into the $Y$-axis input, the instrument corresponds to Tolansky's spectrometer and reproduces the Airy pattern on the scope screen.

The number of interference rings displayed on the screen increases as the a-c voltage is increased (fig. 7). A d-c voltage is superimposed to conveniently center the pattern about a dark or bright ring, as desired (fig. 8). Any need for mechanical positioning of the etalon is thus eliminated.

The a-c voltage was taken from the 60-cycle power line, and was varied with a Variac transformer. The d-c voltage was supplied by the $410 \mathrm{v}$ power supply, or batteries, and was varied with potentiometers. $A$ cathode follower was used between the 1P21 photomultiplier tube and the oscilloscope to provide an impedance match.

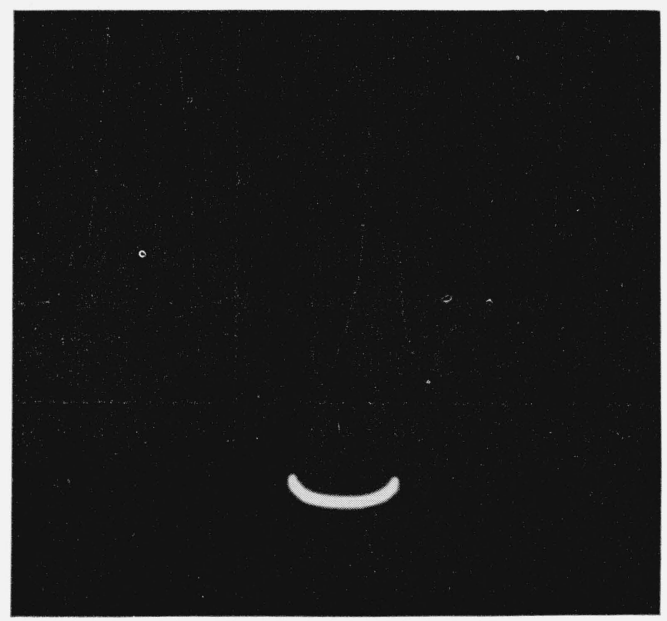

a

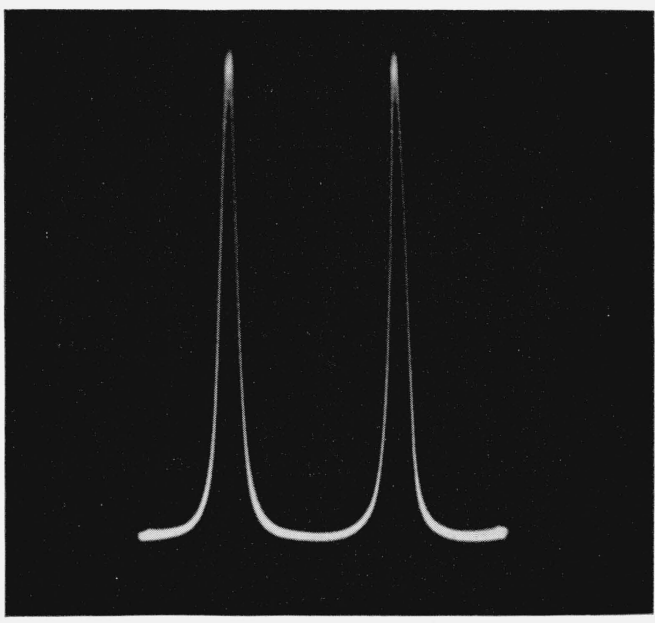

b

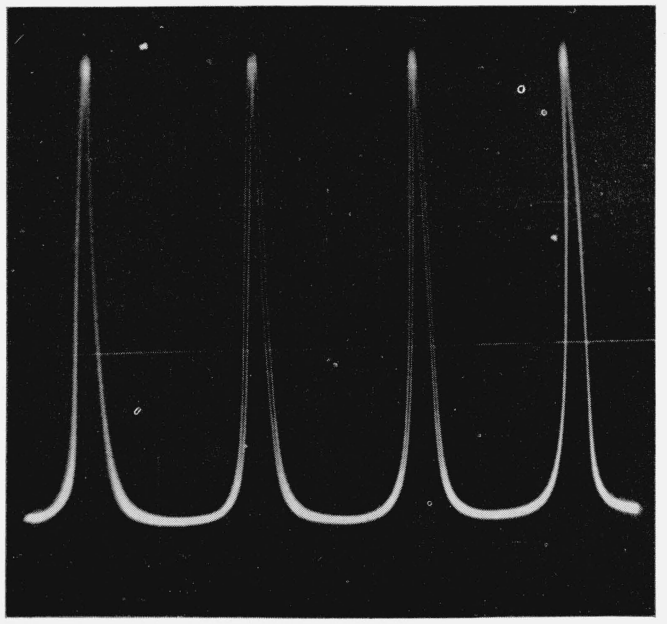

C

Figure 7. Effects of a-c voltage on scope pattern. (a) 50 volts, (b) 150 volts, (c) 250 volts rms. 

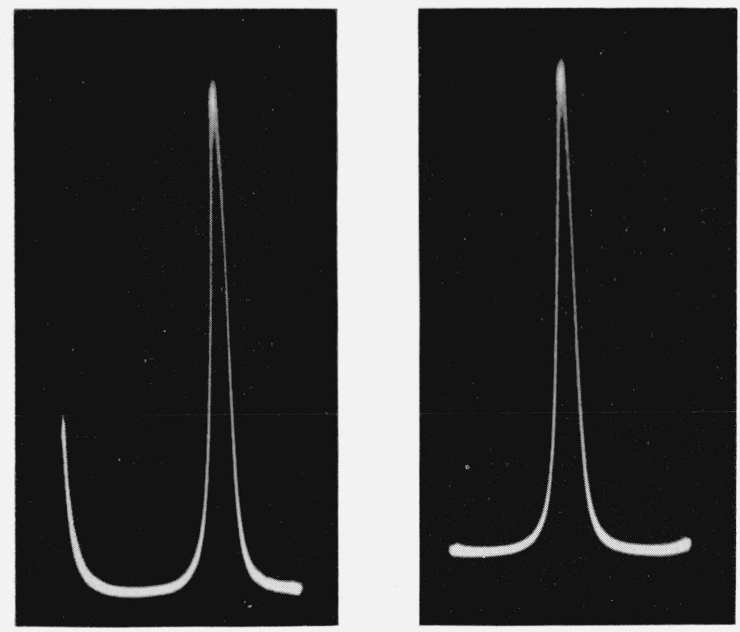

a

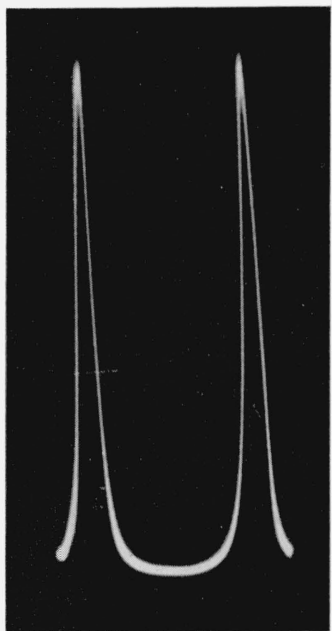

C

Figure 8. Effect of $d$-c voltage on scope pattern. (a) 0 volts, (b) 40 volts, (c) 130 volts.

The oscillograms shown in figures 7 and 8 were obtained with the $8-\mathrm{mm}$ etalon, illuminated with a Spectra-Physics Model $115 \mathrm{He}-\mathrm{Ne}$ laser operating at $6328 \AA$. The resolving power of the etalon was not sufficient to resolve the laser modes.

With the 10-cm etalon, however, the patterns shown in figure 9 were obtained in which the modes are clearly resolved. As the laser used was not designed for frequency stability, the oscilloscope displayed a continuously changing pattern. The series of pictures in figure 9 were selected at random to show this effect. It is almost impossible, though, to reproduce in print this very striking demonstration of how, in such a laser, energy continuously shifts back and forth between modes.

Only an oscilloscope can display such rapid changes, and here, then, lies the advantage of the oscillating Fabry-Perot spectrometer as compared to the recording one.
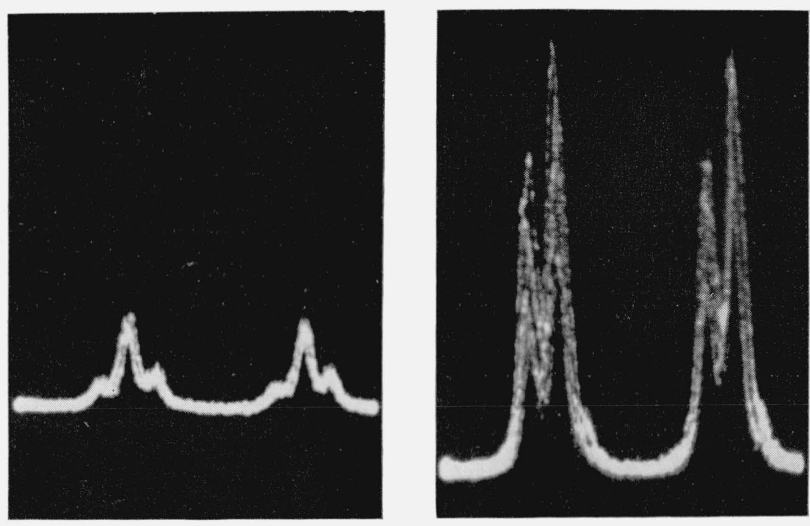

a

b

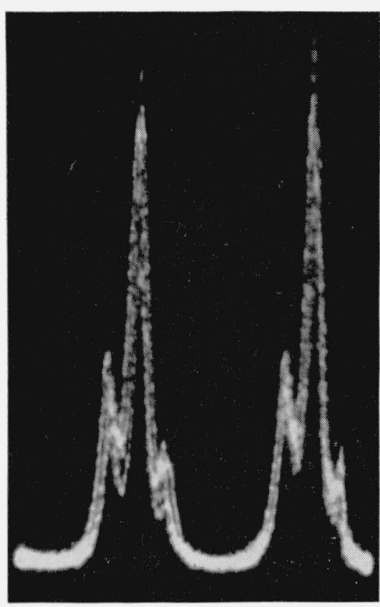

C

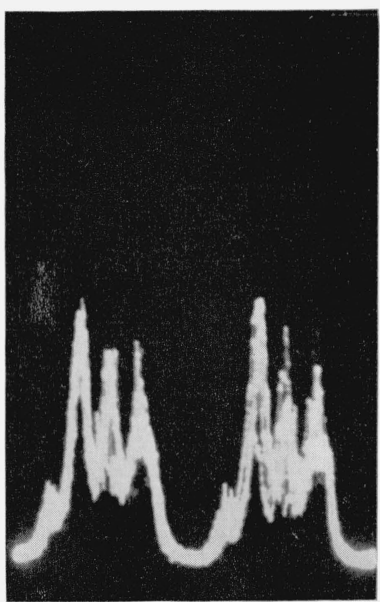

d

FIGURE 9. Varying frequency output of He-Ne laser at $6328 \mathrm{~A}$ 10-cm etalon.

\section{Conclusion}

A preliminary setup only, with much room for improvement, was used for the above described experiments. Yet, a simple Fabry-Perot spectrometer with a "fixed" piezoelectric spacer has proved to be a most adaptable and useful spectrum analyzer for high-resolution spectroscopy and laser work. It is planned to use such spectrometers, in an improved, permanent design, for the analysis of the frequency output of infrared and visible lasers. For this purpose, greater spacings than the ones used here will be required to provide better resolution.

The use of piezoelectric spacers to tune laser cavities has also been realized in this laboratory.

K. E. Gillilland and T. Morokuma, of this laboratory, provided valuable ideas and skillful assistance throughout the work described. H. D. Cooke and 
J. J. Spijkerman, both at NBS, made helpful suggestions. The loan of the gas laser by R. L. Mortensen, of Spectra-Physics, is greatly appreciated.

\section{References}

[1] P. Jacquinot and C. Dufour, J. Rech. Centre Nat. Rech. Sci. 2, 91 (1948).

[2] A. Steudel, Naturwiss. 44, 249 (1957).
[3] S. Tolansky and D. J. Bradley, Nat. Phys. Lab. Symp. 11., Interferometry, Her Majesty's Stationary Office, p. 375 (London, 1960).

[4] D. R. Herriot, Appl. Opt. 2, 865 (1963).

[5] C. Candler, Modern Interferometers, Hilger \& Watts, p. 213 (London, 1951).

[6] R. Dupeyrat, Colloq. Intern. Centre Nat. Rech. Sci. LXXX, p. 106 (Pans, 1958).

(Paper 68C1-147) 